\title{
La dosimétrie et spectrométrie neutroniques : la contribution de l'université autonome de Barcelone
}

\author{
F. FERNÁNDEZ ${ }^{1}$, T. BOUASSOULE ${ }^{1}$, M. TOMÁS $^{1}$, K. AMGAROU $^{1}$, \\ C. DOMINGO ${ }^{1}$, M.J. GARCÍA ${ }^{1}$, J. CASTELO ${ }^{1}$
}

(Manuscrit reçu le 6 novembre 2006, accepté le 28 février 2007)

RÉSUMÉ Depuis la création de l'université autonome de Barcelone, notre groupe a mené une recherche à la fois théorique et expérimentale sur les rayonnements cosmiques et leur détection au moyen de détecteurs passifs. La connaissance acquise sur le processus d'enregistrement des détecteurs solides de traces et les caractéristiques de ces détecteurs ont permis au groupe d'étendre sa recherche vers d'autres applications et d'autres types de détecteurs. Nous présentons nos travaux principaux de recherche sur ce thème en prenant en compte les résultats les plus significatifs dans cette étude.

ABSTRACT Neutron dosimetry and spectrometry: contribution of Universitat Autònoma de Barcelona.

From the creation of the Universitat Autònoma de Barcelona, our group has carried out both experimental and theoretical research related to Cosmic Rays and their detection by means of passive detectors. The knowledge acquired on the process of ion registration in Solid State Nuclear Track Detectors and on the detector characteristics, has allowed the Group to extend the research to other applications and other types of detectors. The more relevant research lines corresponding to this subject and performed in our group are presented taking into account the more relevant results achieved in this study.

Keyword: neutron dosimetry / neutron spectrometry / SSNT detectors

\section{Introduction: présentation et développement historique de nos détecteurs}

Depuis la création de l'université autonome de Barcelone (1970), notre groupe G.F.R. (Grup de Física de les Radiacions) a porté à terme une recherche expérimentale et théorique dans le domaine des rayons cosmiques et de leur détection au moyen de détecteurs passifs. La connaissance acquise sur le processus d'enregistrement d'ions dans les détecteurs solides de traces nucléaires et leurs caractéristiques a permis à notre groupe d'étendre la recherche à d'autres applications et à d'autres types de détecteurs.

\footnotetext{
${ }^{1}$ Grup de Física de les Radiacions, Departament de Física, Universitat Autònoma de Barcelona, 08193 Bellaterra, Espagne.
} 
Les axes de recherche développés dans ce domaine sont les suivants :

- la dosimétrie des neutrons avec les détecteurs solides de traces nucléaires (SSTN) ;

- la dosimétrie des neutrons avec les détecteurs à semi-conducteurs ;

- la spectrométrie des neutrons avec les sphères de Bonner.

\section{Le détecteur de traces CR-39 : son optimisation comme détecteur pour la dosimétrie individuelle des neutrons}

Notre activité principale a porté sur l'étude et le calcul par simulation de l'interaction des neutrons avec la matière produisant des particules chargées et des gamma qui peuvent être détectés par ces détecteurs via les particules secondaires chargées.

De 1988 à 1992, les recherches ont consisté à développer un système basé sur le CR-39 et à étudier son développement électrochimique et son efficacité (Fernández et al., 1988), ce qui nous a permis de trouver une fonction semiempirique pour l'efficacité de la détection des protons par le CR-39 en fonction de l'énergie et de l'angle d'incidence.

La première optimisation du dosimètre a été faite en 1991 (Fernández et al., 1991), avec une configuration constituée d'un convertisseur de polyéthylène et d'un détecteur CR-39. L'optimisation a consisté à étudier plusieurs épaisseurs de polyéthylène avec l'objectif d'arriver à un équilibre protonique, pour améliorer la réponse du détecteur CR-39 aux neutrons. L'influence de différentes épaisseurs du détecteur CR-39 a elle aussi été étudiée. La réponse du dosimètre présentait une réponse non plane, avec une forte dépendance de l'énergie (Fernández et al., 1992). Dans le but d'améliorer la réponse du dosimètre aux neutrons rapides, une nouvelle configuration a été proposée, ajoutant une épaisseur de Makrofol de $300 \mathrm{~mm}$ entre le convertisseur de polyéthylène et le détecteur CR-39, l'épaisseur du Makrofol se comportant à la fois comme convertisseur de neutrons et comme détecteur des particules chargées lourdes. Les premiers résultats avec cette configuration ont été publiés en 1996 (Fernández et al., 1996) et développés encore plus tard par une étude et une caractérisation complète du dosimètre avec une optimisation du système de développement électrochimique, du CR-39, du Makrofol et du système de lecture automatique des traces (Bouassoule, 1988). Une étude expérimentale et théorique exhaustive de la réponse du dosimètre aux champs de neutrons monoénergétiques et réalistes nous a permis d'obtenir une réponse assez plane dans la zone des neutrons rapides mais pas assez dans la zone des neutrons thermiques. D'où la nécessité de développer une nouvelle configuration pour améliorer la réponse du dosimètre non seulement pour les neutrons rapides mais surtout pour les neutrons thermiques. 
TABLEAU I

Facteur d'étalonnage (géométries ISO et ROT) pour le spectre neutronique à l'intérieur du bâtiment réacteur de la centrale nucléaire de Vandellòs II et pour les sources de référence ${ }^{252} \mathrm{Cf}$, ${ }^{241}$ Am et l'empilement thermique SIGMA (géométrie A-P) de Cadarache.

Calibration factor (ISO and ROT geometries) for the neutron spectra inside the containment building of the nuclear power plant of Vandellòs II, as well as for the reference sources, ${ }^{252} \mathrm{Cf}$, ${ }^{241} \mathrm{Am}$ and the SIGMA thermal source (A-P geometry) of Cadarache facilities.

\begin{tabular}{lccc}
\hline \multicolumn{4}{c}{ Facteur d'étalonnage } \\
\hline & $\mathrm{Hp}(10,0) *(\mathrm{~Sv})$ & Dose efficace (Sv) & \\
Spectre & PADC0 & PADC0 & Géometries \\
Thermalisé & 14,7 & 12,2 & BELCH \\
Thermalisé & 14,7 & 5,6 & ISO \\
${ }^{252} \mathrm{Cf}$ & 4,0 & 4,0 & AP \\
${ }^{241} \mathrm{Am}-\mathrm{Be}$ & --- & --- & AP \\
SIGMA & 7,3 & 11,8 & AP \\
\hline
\end{tabular}

*: Hp(10,0) est l'équivalent de dose individuel pour une incidence normale.

Comme cette dernière configuration n'était pas sensible aux neutrons thermiques, un nouveau convertisseur a été ajouté entre le Makrofol et le détecteur CR-39 (Bouassoule et al., 1999). Il s'agit d'un convertisseur de $3 \mathrm{~mm}$ d'air, qui convertit les neutrons thermiques en protons par l'intermédiaire de la réaction ${ }^{14} \mathrm{~N}$ $(\mathrm{n}, \mathrm{p}){ }^{14} \mathrm{C}$. Cette configuration (PADC0) a été exposée dans différents points du bâtiment réacteur de la centrale nucléaire de Vandellòs II (Fernández et al., 2004a) durant une campagne de caractérisation des spectres de neutrons avec notre système de spectrométrie neutronique par sphères de Bonner.

L'étalonnage des dosimètres de routine est généralement fait en exposant ceuxci à des sources de neutrons de référence. Le facteur d'étalonnage obtenu pour cette configuration (PADC0) indique que la réponse du dosimètre aux neutrons thermiques est encore nettement faible. Cependant, cette conclusion nous a amenés à penser à d'autres configurations pour améliorer la réponse du dosimètre aux neutrons thermiques. Les valeurs trouvées pour ces facteurs d'étalonnage montrent que l'usage commun de dosimètres individuels de neutrons étalonnés avec une source standard ISO (comme par exemple le ${ }^{252} \mathrm{Cf}$ et ${ }^{241} \mathrm{Am}$ ), n'est pas approprié pour les spectres réalistes qu'on peut rencontrer dans l'industrie nucléaire.

Durant les trois dernières années, le travail du GFR a été orienté en vue d'obtenir un dosimètre individuel de neutrons basé sur le CR-39 comme détecteur et plusieurs convertisseurs capables d'estimer la dose dans des champs de neutrons thermalisés, comme ceux trouvés dans les centrales nucléaires. Les deux nouvelles 


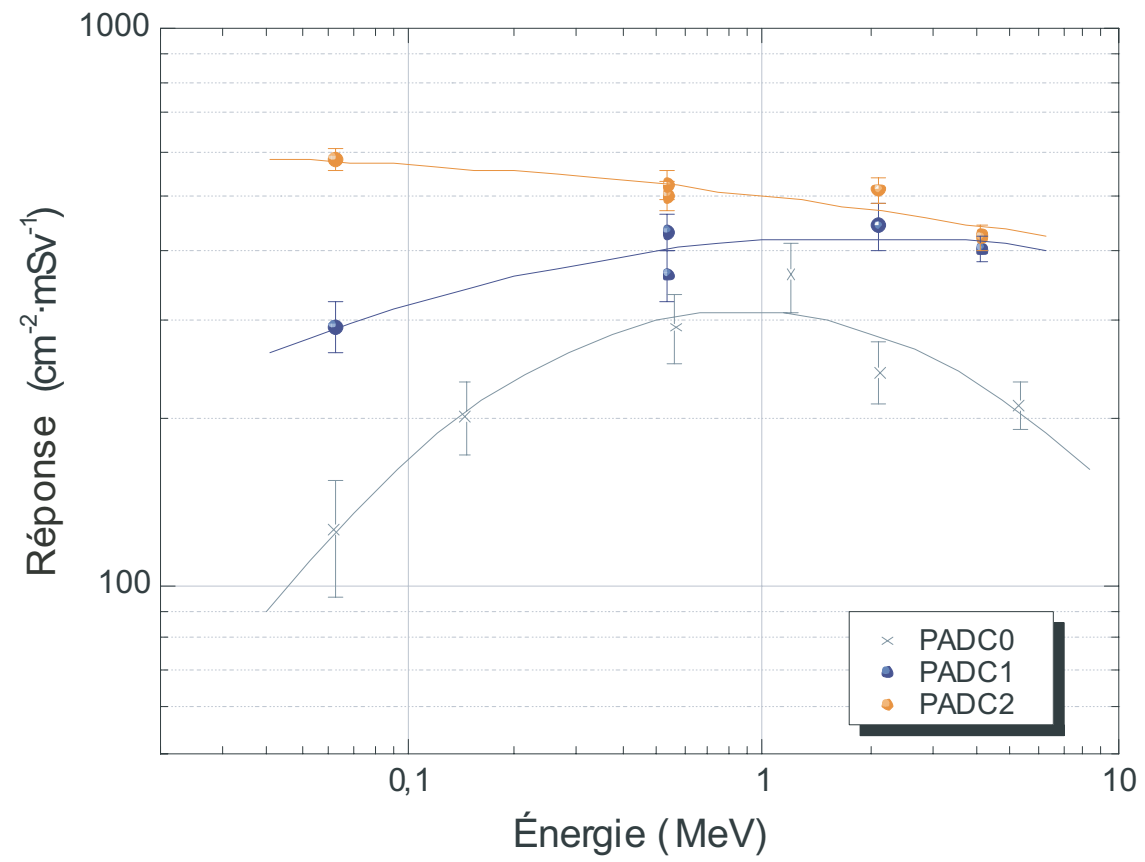

Figure 1 - Réponse en fonction de l'énergie des neutrons pour les trois configurations étudiées. Response as a function of the neutron energy for the three studied configurations.

configurations étudiées par notre groupe ont consisté à élever le pourcentage d'azote ${ }^{14} \mathrm{~N}$ dans les convertisseurs pour les neutrons thermiques. La première est composée d'un convertisseur en air de $6 \mathrm{~mm}$ d'épaisseur (PADC1) et la deuxième d'un convertisseur en nylon de $100 \mu \mathrm{m}$ d'épaisseur (PADC2); cette dernière configuration a montré une amélioration très notable de la réponse du dosimètre aux neutrons thermiques.

Comme on peut l'observer dans la figure 1, la configuration PADC2 constitue une avancée importante dans la réponse de ce dosimètre en vue de son application en dosimétrie individuelle des neutrons dans une large zone d'énergie.

Nous avons publié dans ce domaine un total de dix-sept articles dans des journaux internationaux, dix dans des journaux nationaux, cinq travaux de recherches, deux thèses d'état et nous avons participé à plusieurs programmes de recherche. 


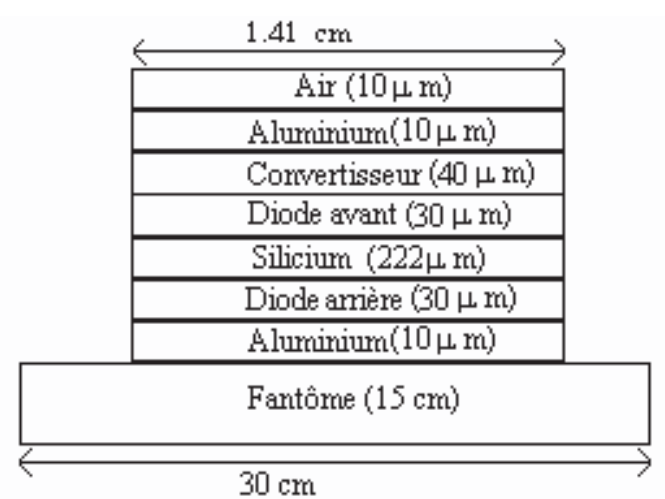

Figure 2 - Coupe synoptique du dosimètre utilisé pour la simulation. Real geometry of dosemeter used for simulation purposes.

\section{La dosimétrie individuelle des neutrons avec les détecteurs semi-conducteurs}

Dans le cadre d'un projet européen, notre groupe, durant les années 1993-1995, a étudié par simulation avec le code MCNP4A les possibilités de concevoir un dosimètre composé d'une double diode (CD-NEUT-200-DBL) et d'un convertisseur de polyéthylène dans le but de l'utiliser en dosimétrie individuelle des neutrons rapides. Cette étude nous a permis de mettre en évidence l'impossibilité de séparer les composantes $n-\gamma$ pour des énergies de neutrons inférieures à $350 \mathrm{keV}$ et la difficulté d'interpréter la réponse quand les neutrons génèrent dans le convertisseur des protons avec des énergies suffisantes pour traverser la diode. Dans l'intervalle $400 \mathrm{keV}-1,5 \mathrm{MeV}$, nous avons trouvé qu'il n'est pas nécessaire d'utiliser la méthode différentielle pour séparer les composantes $n-\gamma$.

Le dosimètre, composé de huit matériaux différents (Fig. 2), air, sachet d'aluminium, convertisseur de polyéthylène $(n, p)$, double diode, est finalement posé sur un fantôme de méthacrylate $(30 \times 30 \times 15) \mathrm{cm}^{3}$. Le détecteur est constitué de deux diodes, avec une surface utile de $2 \mathrm{~cm}^{2}$, localisées sur les deux côtés opposés d'un bloc de silicium (avant, arrière). La zone désertée pour une polarisation de $10 \mathrm{~V}$ est de $30 \mu \mathrm{m}$ pour chacune des deux diodes et la séparation entre elles est de $222 \mu \mathrm{m}$ de silicium. Des préamplificateurs Ortec, des amplificateurs et un analyseur multi canal Canberra ont été utilisés avec un ordinateur portable et un logiciel Maestro II (Ortec) pour l'acquisition des 
données. Le dosimètre, une fois étalonné, a été soumis à un ensemble d'irradiations, dans le cadre d'une campagne d'intercomparaison qui a eu lieu dans le Centre nucléaire de Bruyères-le-Châtel. Les résultats expérimentaux obtenus et leurs analyses ont été publiés en 1993 (Fernández et al., 1993).

Pour simuler l'interaction des neutrons avec le dosimètre, nous avons utilisé le code MCNP4A (Briesmeister, 1993) avec la géométrie décrite précédemment, ce qui nous a obligé, étant donné la position des différents matériaux qui entourent le détecteur, à définir 17 plans qui limitent les zones ou régions où se trouve chacun de ces matériaux.

Dans le cas des particules chargées et pour les énergies des neutrons utilisées dans ce travail, on considère seulement les collisions élastiques et inélastiques avec les noyaux du convertisseur. Puisque le code MCNP4A ne considère pas dans cette version le transport des protons formés dans le convertisseur, nous avons utilisé le code PROT développé par notre groupe (Fernández et al., 1992).

Des faisceaux monoénergétiques de $73 \mathrm{keV}, 144 \mathrm{keV}, 250 \mathrm{keV}, 570 \mathrm{keV}$, 1,2 MeV et 2,5 MeV, avec une incidence normale, ont été utilisés pour la simulation. Les résultats obtenus confirment ce qu'on attendait, à savoir une présence importante de photons et d'électrons liés à la présence du fantôme et le fait que les photons observés proviennent dans la grande majorité de l'interaction des neutrons avec l'hydrogène du fantôme. Par ailleurs, les impulsions des photons produits dans les deux diodes ne dépassent pas l'énergie de $25 \mathrm{keV}$ et la différence entre les spectres d'impulsions correspondants aux deux diodes est très petite en valeur absolue, mais systématiquement négative. Ces caractéristiques du spectre différentiel, obtenues par différence entre les diodes, nous ont amenés à considérer dans des calculs postérieurs le spectre de la diode avant, au lieu du différentiel, puisque de cette manière nous évitons la difficulté qu'engendre le traitement de spectres avec des contenus négatifs.

À partir des résultats obtenus par simulation, nous pouvons considérer que les contributions les plus importantes à la formation des impulsions dans la diode avant proviennent des photons générés dans le fantôme et des protons générés dans le convertisseur. En tenant compte de cette circonstance et en admettant que le spectre généré par les photons a un comportement exponentiel en fonction de l'énergie, nous avons ajusté par la méthode du $\chi^{2}$ les spectres expérimentaux (diode avant) correspondants aux énergies de $570 \mathrm{keV}, 1,2 \mathrm{MeV}$ et 2,5 MeV (pour les autres énergies nous n'avons pas obtenu de résultats valables) avec une fonction définie par l'expression :

$$
F_{i}=\left[\frac{M C_{i}}{\sum M C_{i}} \alpha+(1-\alpha) \frac{e^{-\beta E_{i}}}{\sum e^{-\beta E_{i}}}\right] N
$$




\section{TABLEAU II}

Résultat des ajustements et leur comparaison avec les données expérimentales. Fits results and their comparison with the experimental ones.

\begin{tabular}{lccc}
\hline Energie des neutrons $(\mathrm{MeV})$ & 0,570 & 1,2 & 2,5 \\
\hline Ajustement : & 8 & 13 & 17 \\
$\begin{array}{l}\text { Degré de liberté: } \\
\text { Valeur de } \chi^{2}:\end{array}$ & 7 & 15 & 26 \\
$\begin{array}{l}\text { Valeur ajustée de } \alpha \\
\text { Valeur ajustée de } \beta\end{array}$ & $0,350 \pm 0,025$ & $0,71 \pm 0,025$ & $0,893 \pm 0,030$ \\
Nombre de protons détectés & $-0,0129 \pm 0,0040$ & $-0,0115 \pm 0,005$ & $-0,0120 \pm 0,005$ \\
Extrapolation à $0 \mathrm{keV}$ : & $3199 \pm 278$ & $2319 \pm 95$ & $12213 \pm 515$ \\
$\begin{array}{l}\text { Nombre de protons produits } \\
\text { dans le convertisseur }\end{array}$ & $39989 \pm 3475$ & $9465 \pm 388$ & $24279 \pm 1022$ \\
$\begin{array}{l}\text { Fluence de neutrons calculée } \\
\text { (neutron cm }\end{array}$ & $(11,00 \pm 0,95) \times 10^{6}$ & $(3,94 \pm 0,16) \times 10^{6}$ & $(15,00 \pm 0,63) \times 10^{6}$ \\
$\begin{array}{l}\text { Dose }(\mathrm{mSv}): \\
\begin{array}{l}\text { Calculée } \\
\text { Expérimentale }\end{array}\end{array}$ & $3,75 \pm 0,32$ & $1,64 \pm 0,07$ & \\
\hline
\end{tabular}

où $\mathrm{F}_{\mathrm{i}}$ est le nombre d'impulsions calculé pour un intervalle d'énergie autour de $\mathrm{E}_{\mathrm{i}}$, normalisé au nombre total d'impulsions enregistrées $\mathrm{N}, \mathrm{MC}_{\mathrm{i}}$ le nombre d'impulsions correspondant aux protons d'énergie $\mathrm{E}_{\mathrm{i}}$ calculé avec le code PROT et $\alpha$ et $\beta$ deux paramètres à ajuster, qui représentent le pourcentage des protons dans le spectre total et la constante de la fonction exponentielle avec laquelle on décrit le comportement des impulsions dues aux photons.

La méthode d'ajustement utilisée pour ces deux énergies était insuffisante pour les neutrons d'énergie 2,5 MeV, étant donné que pour cette valeur, on produit des protons avec une énergie suffisante pour traverser la diode et, dans de telles circonstances, comme nous le savons, les diodes n'ont pas une réponse facile à interpréter (Knoll, 1989). Malgré ces circonstances, nous avons essayé d'ajuster le spectre de 2,5 MeV, altérant dans le code PROT l'énergie du proton, choisi aléatoirement dans une gaussienne, dont la valeur moyenne correspond à celle déduite par accomplissement des conditions cinématiques imposées dans sa génération, moyennant une déviation qui au lieu d'être donnée par la théorie classique de Bhor (Segre, 1977), est donnée par la théorie de Tschalär (Tschalär and Maccabee, 1977) plus une quantité calculée par l'expression suivante :

$$
\frac{\Delta \mathrm{E}_{\mathrm{i}}}{\mathrm{E}_{\mathrm{i}}}=\delta\left(\mathrm{E}_{\mathrm{i}}\right)
$$

où $\Delta \mathrm{E}_{\mathrm{i}}$ est l'incertitude assignée à l'énergie du proton et $\delta\left(\mathrm{E}_{\mathrm{i}}\right)$ un coefficient à déterminer pour chaque valeur de $\mathrm{E}_{\mathrm{i}}$. Dans le tableau II, nous présentons également les résultats les plus significatifs avec les données de l'accélérateur dans 
le but de valider les ajustements réalisés. L'accord est correct pour les deux premières valeurs d'énergie et ne l'est pas pour 2,5 MeV.

En conclusion de cette étude, nous avons pu constater que la réponse du dosimètre n'est pas correcte pour des énergies des neutrons qui produisent des protons avec des énergies suffisantes pour le traverser (1,525 MeV dans notre cas), si bien que cette limite peut être élargie en augmentant son épaisseur. Par ailleurs, dans l'intervalle d'utilisation $(0,400<\mathrm{E}<1,525 \mathrm{MeV})$, il n'est pas nécessaire d'utiliser la méthode différentielle pour séparer les composantes neutron gamma, étant donné qu'on peut la réaliser par calcul.

Nous avons publié dans ce domaine un total de trois articles dans les journaux internationaux, trois dans des journaux nationaux, un travail de recherche et nous avons participé à un programme de recherche.

\section{La spectrométrie et dosimétrie des neutrons avec un système de sphères de Bonner}

\subsection{Introduction}

Les neutrons constituent un rayonnement indirectement ionisant qui produit pour la même dose absorbée des effets biologiques plus importants que ceux produits par les photons. La dosimétrie des neutrons a dû surmonter de nombreuses difficultés au cours de son développement durant ces dernières décennies.

Actuellement les instruments de radioprotection opérationnelle utilisés pour la dosimétrie des neutrons ont des réponses très dépendantes de la distribution d'énergie du spectre des neutrons et principalement de la présence d'une composante importante de neutrons d'énergie intermédiaire. Pour cette raison, il devient difficile d'interpréter les indications de ces instruments si on ne connaît pas la distribution spectrale de la fluence neutronique aux points de mesure (Muller et al., 2002).

Étant donné que cette distribution spectrale peut elle-même changer à l'intérieur de chaque installation nucléaire d'une manière appréciable, la spectrométrie des neutrons est d'un intérêt primordial pour l'étalonnage correct des instruments de mesure et l'évaluation précise des grandeurs dosimétriques. Elle est aussi nécessaire pour l'élaboration des protocoles et stratégies appropriés pour la protection radiologique.

\subsection{Spectrométrie par la méthode des multisphères}

Le spectromètre multisphères, aussi appelé système de sphères de Bonner (BSS), a été décrit pour la première fois en 1960 par Bramblett, Erwing et Bonner 
(Bramblett et al., 1960). Ce système est constitué par un détecteur de neutrons thermiques $\left({ }^{6} \mathrm{Li},{ }^{10} \mathrm{~B}\right.$ ou $\left.{ }^{3} \mathrm{He}\right)$, enveloppé par des sphères modératrices de différents diamètres, souvent de polyéthylène pour que ces détecteurs soient sensibles aux neutrons de plus haute énergie. Les neutrons rapides sont freinés à l'intérieur du modérateur et arrivent au détecteur à l'état thermique tandis que les neutrons initialement thermalisés sont partiellement capturés à l'intérieur du modérateur et n'atteignent pas le détecteur.

Le taux de comptage $\mathrm{M}_{\mathrm{dr}}$ (count/s), obtenu avec une sphère de rayon $\mathrm{r}$ dans un point d'un champ neutronique donné, est la convolution de la fonction réponse $\mathrm{R}_{\mathrm{r}}(\mathrm{E})\left(\mathrm{cm}^{2}\right)$ de cette sphère, avec le taux de la distribution de la fluence différentielle en énergie $\dot{\Phi}_{\mathrm{E}}(\mathrm{E})\left(\mathrm{MeV}^{-1} \mathrm{~cm}^{-2} \mathrm{~s}^{-1}\right)$ des neutrons mesurés dans ce même point. L'utilisation de plusieurs sphères de différents rayons dans un champ de neutrons inconnu conduit à un système d'équations :

$$
\mathrm{M}_{\mathrm{dr}}=\sum_{\mathrm{j}=1}^{\mathrm{n}} \mathrm{F}_{\mathrm{rj}} \dot{\Phi}_{\mathrm{E}}\left(\mathrm{E}_{\mathrm{j}}\right) \Delta \mathrm{E}_{\mathrm{j}} ; \mathrm{r}=1, \ldots, \mathrm{m}
$$

$\mathrm{m}$ étant le nombre de sphères utilisées et $\mathrm{n}$ le nombre de groupes d'énergie qui s'étend de l'énergie thermique jusqu'à quelques dizaines de $\mathrm{MeV} . \dot{\Phi}_{\mathrm{E}}\left(\mathrm{E}_{\mathrm{j}}\right) \Delta_{\mathrm{j}}$ est le taux de fluence énergétique des neutrons dans l'intervalle $\Delta \mathrm{E}_{\mathrm{j}}$ et $\mathrm{F}_{\mathrm{rj}}$ la réponse moyenne de la sphère $\mathrm{r}$ dans l'intervalle d'énergie $\mathrm{j}$. La résolution d'un tel système est portée à terme par une procédure de calcul appelée déconvolution. Dans notre cas, cette déconvolution est réalisée avec un code nommé MITOM (Tomás et al., 2004).

Pour la détermination des fonctions réponses, sachant que le nombre d'énergies disponibles est très réduit, il nous a fallu simuler le système de mesure pour évaluer sa réponse, en le validant plus tard avec des énergies de référence.

Le système de multisphères de l'UAB (Bakali, 2001) est composé de huit sphères de polyéthylène, de diamètres $2,5,3,4,2,5,6,8,10$ et 12 pouces (Centronic, 1998). Les trois plus petites sphères sont aussi utilisées avec une coquille de cadmium $(\mathrm{Cd})$ de $1 \mathrm{~mm}$ d'épaisseur. Le détecteur des neutrons utilisé avec les sphères est un compteur proportionnel cylindrique de ${ }^{3} \mathrm{He}$, du type 0.5NH1/1KI fabriqué par Eurisys Mesures (Eurisys, 1998). Les fonctions réponses de ce spectromètre ont été simulées par MCNP4B (Briesmeister, 1997) et validées au PTB (Allemagne) et IRSN (Cadarache, France), laboratoires de référence neutronique pour des faisceaux monoénergétiques et sources ISO (Lacoste et al., 2004). Dans la figure 3 nous présentons les fonctions réponses de notre système obtenues par simulation et validées pour certaines énergies dans des laboratoires de référence neutronique. 


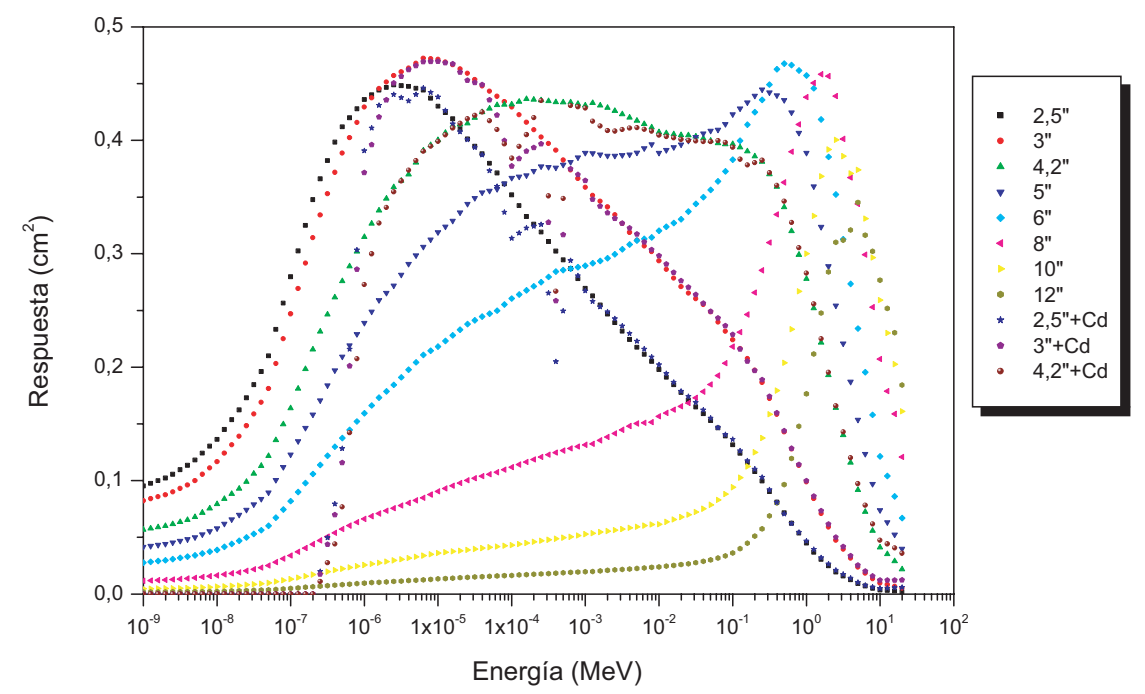

Figure 3 - Fonctions réponses de notre système dosimétrique obtenues par simulation, validées pour différentes énergies dans des laboratoires de référence neutronique.

Response functions for our neutron spectrometer obtained by simulation and validated for several energies in neutron reference laboratories.

\section{Mesures des spectres neutroniques}

Une fois fixées les conditions d'étalonnage du système, les fonctions réponses validées pour chaque point de mesure, nous procédons à la réalisation des mesures aux points sélectionnés. En chaque point, les sphères (huit sans cadmium et trois avec cadmium) avec le détecteur localisé au centre sont posées sur un tripode à une distance du sol prise comme référence de 140 centimètres par rapport au centre du détecteur.

La détermination du spectre neutronique passe par la résolution de l'équation (3) à partir de la déconvolution du spectre mesuré, du taux de distribution de fluence énergétique, de la connaissance des fonctions réponse $\mathrm{F}_{\mathrm{r}}(\mathrm{E})$ et de l'ensemble des mesures $\mathrm{M}_{\mathrm{dr}}$ réalisées.

Dans le but de valider notre système spectrométrique (BSS + MITOM) nous avons participé à un exercice international d'intercomparaison (Gressier et al., 2004) qui a eu lieu à l'IRSN de Cadarache en 2002, sur un spectre réaliste de neutrons (canal/T400). 


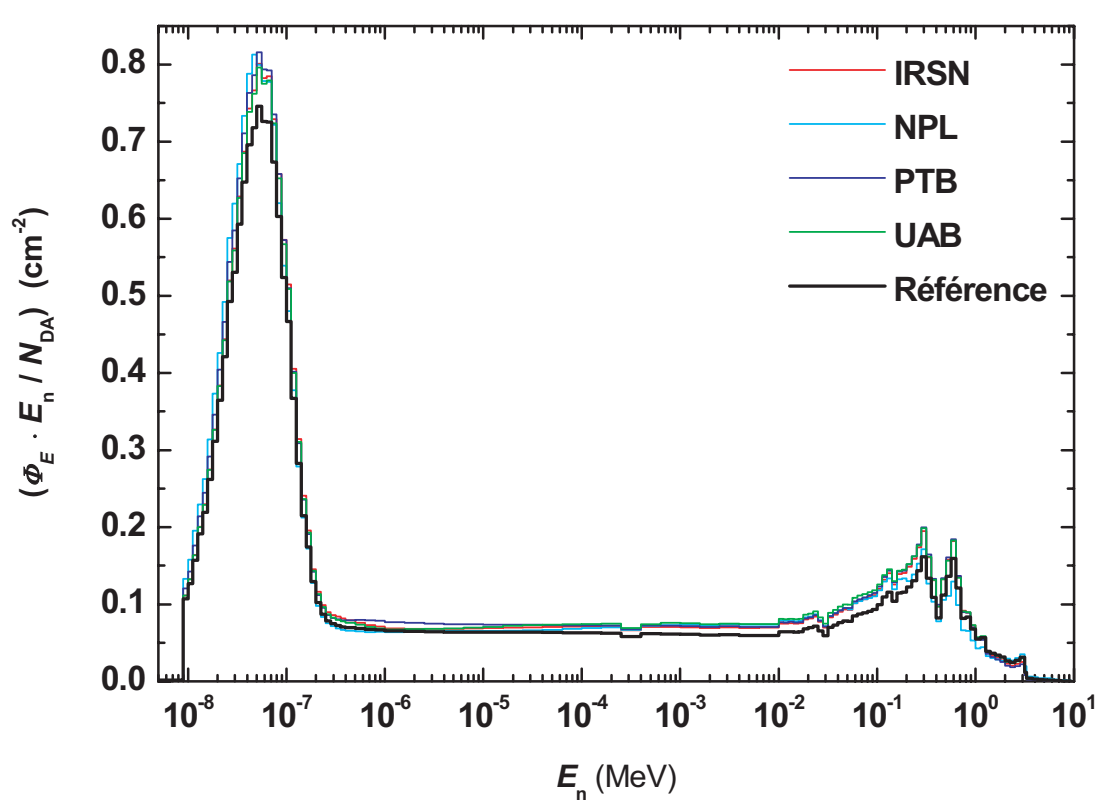

Figure 4-Spectres obtenus par les différents groupes participant à l'exercice international d'intercomparaison (Canel/T400).

Result of different groups for the international intercomparison exercise at Cadarache facilities (Canel/T400).

Dans la figure 4, nous présentons le spectre obtenu par les différents groupes participants et le spectre de référence pour l'ensemble des mesures réalisées dans ce spectre. Comme on peut l'observer, nos résultats sont aussi bons que ceux obtenus par les autres groupes participants, tous reconnus dans le domaine de la spectrométrie neutronique.

\subsection{Centrales nucléaires}

La contribution des neutrons à la dose individuelle dans les centrales nucléaires est en général faible bien qu'il existe des cas dans lesquels l'intervention à l'intérieur du bâtiment réacteur, quand le réacteur nucléaire est en exploitation, peut engendrer quelques risques d'irradiation en neutrons.

Actuellement, la majorité des instruments opérationnels et des dosimètres individuels utilisés en radioprotection ont des réponses très dépendantes de l'énergie, surtout dans le cas des champs de neutrons avec une composante 
importante d'énergies intermédiaires. L'interprétation des lectures données par ces appareils devient alors difficile s'il n'y a pas une connaissance de la fluence de la distribution spectrale des neutrons aux points d'intérêt.

Compte tenu de cette situation, durant les cinq dernières années, le groupe de Physique des rayonnements de l'université autonome de Barcelone (UAB) en collaboration avec l'Institut de radioprotection et sécurité nucléaire (IRSN) de Cadarache a porté à son terme plusieurs campagnes de mesures dans le cadre d'une action nationale de recherche, dans les centrales nucléaires de Vandellòs II, Ascó I, Ascó II et Cofrentes.

Avec le système de spectrométrie décrit ci-dessus, nous avons caractérisé un total de 17 points à l'intérieur du bâtiment réacteur des centrales nucléaires. Ces points qui sont fréquemment visités par les travailleurs quand le réacteur nucléaire est en opération ont été choisis d'après les indications du chef de service de protection radiologique avec la prise en considération des résultats d'un ensemble de mesures effectuées avec les moniteurs Berthold et Dineutron.

Pour chacun des points sélectionnés et pour chaque sphère, un temps d'acquisition convenable a été choisi pour avoir une incertitude statistique sur la valeur de la lecture d'approximativement $1 \%$. Un total de cinq mesures ont été prises pour chacune des onze configurations (sphère nues + sphères avec $\mathrm{Cd}$ ), à une hauteur de 1,4 m au-dessus du sol. Après mesure, les lectures ont été corrigées pour le temps mort et converti en taux de comptage. Ces taux de comptage ont été normalisés par rapport au taux de comptages donné par un système identique avec une sphère de 4,2 pouces, située près de la cuve du réacteur pour chacun des points sélectionnés, afin de prendre en considération les possibles changements du taux de fluence des neutrons pendant la mesure.

Dans le tableau III, nous résumons les résultats de mesure pour les cinq points sélectionnés à l'intérieur du bâtiment réacteur de la centrale nucléaire de Vandellòs II avec les différentes grandeurs utilisées pour la caractérisation du spectre (Fernández et al., 2004b) (le rapport de mesure : 10/3, l'énergie moyenne, $\mathrm{E}_{\mathrm{m}}$, son facteur de qualité moyen, $\mathrm{Q}$, et le coefficient moyen de conversion $\left.\mathrm{h}_{\Phi}(10)\right)$. Le taux de fluence $\left(\dot{\phi}_{\text {total }}\right)$ et le débit d'équivalent de dose ambiant $[\dot{\mathrm{H}} *(10)]$ sont aussi donnés. Les mesures de $[\dot{\mathrm{H}} *(10)]_{\mathrm{n}}$ ont été réalisées avec le dosimètre électronique Dosicard.

Prenant en considération les grandeurs du tableau III, nous pouvons affirmer que nous avons deux types de spectres différenciés. Un peut être considéré comme de première classe, d'après la classification faite par Aroua (1991) avec une énergie moyenne de $20 \mathrm{keV}$. Ce type de spectre est très thermalisé et se trouve 


\section{TABLEAU III}

Résultats obtenus aux points sélectionnés à l'intérieur du bâtiment réacteur de la centrale nucléaire de Vandellòs II avec les différentes grandeurs utilisées pour la caractérisation du spectre de neutrons.

The measurement results for the selected points at different elevations inside the containment building of Vandellòs II nuclear power plant with the different magnitudes used for the characterisation of the neutron spectra.

\begin{tabular}{|c|c|c|c|c|c|c|c|c|c|}
\hline $\begin{array}{c}\text { Points } \\
\text { sélectionnés* }\end{array}$ & Hauteur & $\begin{array}{l}\text { Ratio } \\
10 " / 3 "\end{array}$ & $\begin{array}{c}\mathrm{E}_{\mathrm{m}} \\
(\mathrm{keV})\end{array}$ & Q & $\begin{array}{c}\mathrm{h}_{\Phi}(10) \\
\left(\mathrm{pSV} \mathrm{cm}{ }^{2}\right)\end{array}$ & $\begin{array}{c}\dot{\phi}_{\text {total }} \\
\left(\frac{\text { neutron }}{\mathrm{cm}^{2} \mathrm{~s}}\right)\end{array}$ & $\begin{array}{c}{[\dot{\mathrm{H}} *(10)]_{\mathrm{n}}} \\
(\mu \mathrm{Sv} / \mathrm{h})\end{array}$ & $\begin{array}{c}{\left[\dot{\mathrm{H}}^{*}(10)\right]_{\gamma}} \\
(\mu \mathrm{Sv} / \mathrm{h})\end{array}$ & $\frac{\left[\dot{\mathrm{H}}^{*}(10)\right]_{\mathrm{n}}}{\left[\dot{\mathrm{H}}^{*}(10)\right] \gamma}$ \\
\hline $\mathrm{K} 2$ & 100,0 & 0,26 & 10 & 5,6 & 18 & $1962 \pm 18$ & $129 \pm 3$ & $150 \pm 23$ & $0,9 \pm 0,1$ \\
\hline $\mathrm{J} 3$ & 100,0 & 0,27 & 14 & 6,3 & 21 & $507 \pm 5$ & $39,1 \pm 0,9$ & $110 \pm 17$ & $0,4 \pm 0,1$ \\
\hline $\mathrm{C} 3$ & 114,5 & 0,33 & 24 & 7,3 & 27 & $588 \pm 4$ & $57 \pm 1$ & $18 \pm 3$ & $3,2 \pm 0,5$ \\
\hline A2 & 114,5 & 0,37 & 26 & 7,6 & 29 & $810 \pm 4$ & $84,0 \pm 0,8$ & $20 \pm 3$ & $4,2 \pm 0,7$ \\
\hline A3 & 114,5 & 0,35 & 31 & 8,1 & 32 & $814 \pm 2$ & $93,6 \pm 0,5$ & $25 \pm 4$ & $3,7 \pm 0,6$ \\
\hline D2 & 114,5 & 0,38 & 33 & 8,3 & 33 & $2594 \pm 11$ & $312 \pm 3$ & $61 \pm 9$ & $5,1 \pm 0,8$ \\
\hline
\end{tabular}

* Les indices 2 et 3 représentent la première et deuxième campagne de mesure.

derrière les blindages de béton de plus de $2 \mathrm{~m}$ d'épaisseur combinés avec les géométries du labyrinthe, comme les points K2 et $\mathrm{J} 3$ qui sont situés à la hauteur 100. La deuxième classe de spectres avec des énergies moyennes entre 30 et $70 \mathrm{keV}$ correspond à des champs de neutrons rencontrés derrière des blindages de béton mince (1-2 m), et plus proche du réacteur, c'est le cas des points A3 et D2. Les points $\mathrm{C} 3$ et A2 peuvent aussi être considérés selon ce dernier type de spectres. Il est intéressant de constater que, dans les environs du circuit primaire d'eau (point D2), le spectre neutron présente une valeur plus haute de l'énergie moyenne, c'est une conséquence de l'absence de blindage latéral due à l'existence des barres du réacteur. Les résultats obtenus pour le reste des centrales nucléaires (Aroua et al., 1994) analysées sont très semblables à ceux présentés dans le tableau III.

Finalement, la figure 5 montre les meilleurs ajustements obtenus par notre code de déconvolution MITOM, pour les différents points sélectionnés à l'intérieur du bâtiment réacteur des centrales nucléaires d'Ascó I et Ascó II.

Nous avons publié dans ce domaine un total de dix articles dans des journaux internationaux, six dans des journaux nationaux, trois travaux de recherches, trois thèses d'état et nous avons participé à plusieurs programmes de recherche.

En conclusion, les mesures spectrométriques neutroniques réalisées dans les centrales nucléaires susmentionnées mettent en évidence la présence de spectres assez thermalisés. Dans le même temps, le spectromètre développé par notre groupe peut être considéré comme un système de référence pour la dosimétrie des 


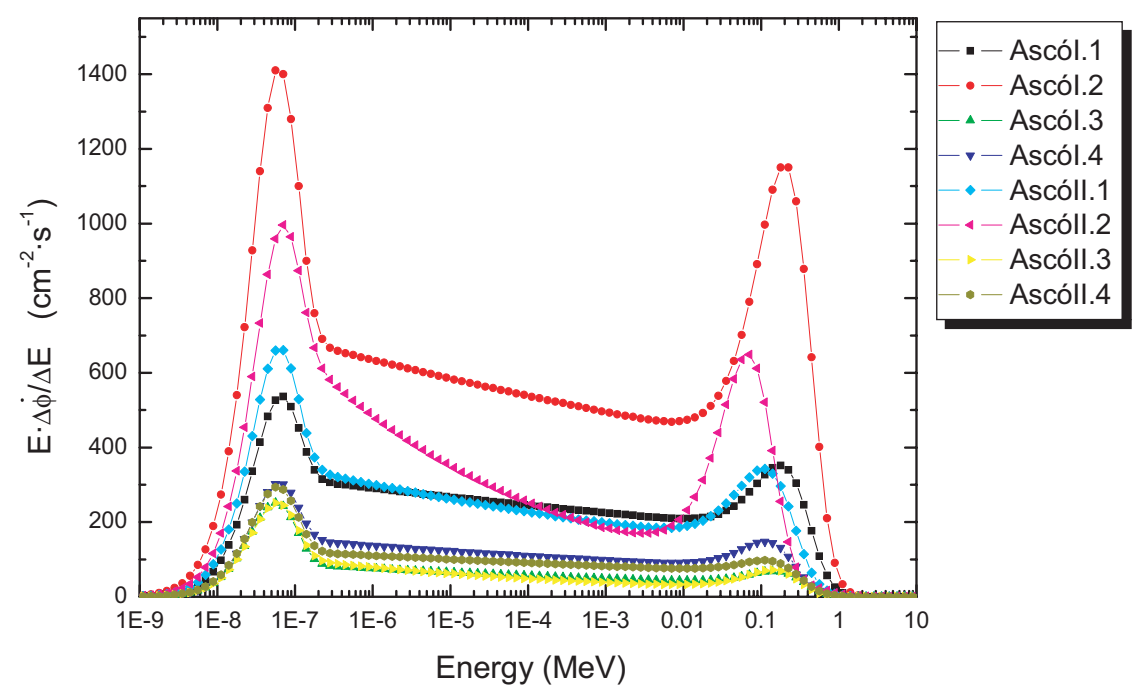

Figure 5 - Spectres de neutrons obtenus par le code MITOM pour les différents points sélectionnés à l'intérieur du bâtiment réacteur des centrales nucléaires d'Ascó I et Ascó II.

Representation of the solution neutron spectra obtained by MITOM code for the different points selected for measurement with the UAB-BSS inside the containment buildings of the Ascó I and Ascó II nuclear power plants.

neutrons compte tenu de sa forte sensibilité qui permet la mesure de bas débits d'équivalent de dose neutron dans des temps raisonnables. Cette information est de grande utilité pour définir une approximation des valeurs de $\dot{H}_{p}(10)$ utilisées pour caractériser un dosimètre individuel des neutrons et définir les protocoles appropriés nécessaires pour un contrôle dosimétrique du personnel adapté à chaque installation nucléaire.

Actuellement, nous travaillons à la mise au point d'un nouveau spectromètre des neutrons adapté aux conditions rencontrées dans les installations d'accélérateurs d'électrons utilisées en radiothérapie.

\section{RÉFÉRENCES}

Aroua A. (1991) Étude des champs neutroniques dans les centrales nucléaires de l'influence de leur diversité sur la détermination des grandeurs de la protection radiologique, Thèse de l'université de Lausanne.

Aroua A., Boschung M., Cartier F., Grecescu M., Prêtre S., Valley J.F., Wernli Ch. (1994) Characterization of the mixed neutron-gamma fields inside the Swiss nuclear power plants by different active system, Radiat. Prot. Dosim. 51, 17-25.

Bakali M. (2001) Espectrometría neutrónica en las centrales nucleares mediante un sistema de esferas Bonner. Thèse de l'université Autonome de Barcelone. 
Bouassoule T. (1988) Contribución a la Dosimetría de Neutrones Por Detectores Sólidos de Trazas. Thèse de l'université Autonome de Barcelone.

Bouassoule T., Fernández F., Marín M., Tomás, M. (1999) A Dosemeter Based on Nuclear Etched Track Detectors for Thermal, Fast and High Energy Neutrons with Flat Response, Radiat. Prot. Dosim. 85(1-4), 39-43.

Bramblett R.L., Ewing R.I., Bonner T.W. (1960) A new type of neutron spectrometer, Nucl. Instr. Meth. 9, 1-12.

Briesmeister J.L. Ed. (1993) MCNP, A général Monte Carlo N-Particle Transport Code, Version 4A, LA-12625

Briesmeister J.F. (1997) MCNP-B. A general Monte-Carlo N-Particle transport code version 4B. Los Alamos National Laboratory. LA-12625-M, New Mexico.

Eurisys (1998), Compteur ${ }^{3} \mathrm{He}$, Notice Technique, Eurisys Mesures.

Fernández F., Baixeras C., Zamani M., López D., Jokic S., Debeauvais M., Ralarosy J. (1988) CR-39 Registration Efficiency of Protons Using Electrochemical Etching, Radiat. Prot. Dosim. 23, 175-178.

Fernández F., Domingo C., Baixeras C., Luguera E., Zamani M., Debeuavais M. (1991) Fast Neutron Dosimetry with CR-39 Using Electrochemical Etching, Nucl. Tracks Radiat. Meas. 19, 467-470.

Fernández F., Domingo C., Luguera E., Baixeras C. (1992) Experimental and Theoretical Determination of the Fast Neutron Response Using CR-39 Plastic Detectors and Polyethylene Radiators, Radiat. Prot. Dosim. 44, 337-340.

Fernández F., Luguera E., Domingo C. Baixeras C. (1993) Separation of the neutron signal from the gamma component in $(\mathrm{n}-\gamma)$ fields using differential pulse analysis techniques with a double silicon diode, Radiat. Prot. Dosim. 70, 87-92.

Fernández F., Bouassoule T., Domingo C., Luguera E., Baixeras C. (1996) Response of a CR-39 Fast Neutron Dosemeter with a Polyethylene Converter Improved with Makrofol, Radiat. Prot. Dosim. 66, 343-347.

Fernández F., Bakali M., Amgarou K., Nourredine A., Mouhssine D. (2004a) Personal Neutron Dosimetry in Nuclear Power Plants Using Etched Track and Albedo Thermoluminiscence Dosemeters, Radiat. Prot. Dosim. 110, 701-704.

Fernández F., Bakali M., Tomas M., Muller H., Pochat J. (2004b) Neutron measurements in the Vandello_s II nuclear power plant with a Bonner Sphere System, Radiat. Prot. Dosim. 110, 517-521.

Gressier V., Lacoste V., Lebreton L., Muller H., Pelcot G., Bakali M., Fernández F., Tómas M., Roberts N.J., Thomas D.J., Reginatto M., Wiegel B., Wittstock J. (2004) Characterisation of the IRSN CANEL/T400 facility producing realistic neutron fields for calibration and test purposes, Radiat. Prot. Dosim. 110, 523-527.

Knoll G.F. (1989) Radiation Detection and Measurement. John Willey \& Sons.

Lacoste V., Gressier V., Pochat J.L., Fernández F., Bakali M., Bouassoule T. (2004) Characterization of Bonner sphere systems at monoenergetic and thermal neutron fields, Radiat. Prot. Dosim. 110, 529-532.

Muller H., Fernández F., Van Ryckeghem L., Alexandre P., Bouassoule T., Pochat J.L., Tomás M. (2002) Monte-Carlo calculations and experimental results of Bonner spheres systems with a new cylindrical Helium-3 proportional counter, Nucl. Instr. Meth. 476, 411-415.

Segre E. (1977) Nuclei and Particles. The Benjamin/Cummings. Publishing Company, Inc.

Tomás M., Fernández F., Bakali M., Muller H. (2004) MITOM: A new unfolding code based on a spectra model method applied to neutron spectrometry, Radiat. Prot. Dosim. 110, 545-548.

Tschalär C., Maccabee H.D. (1977) Energy-Straggling measurement of heavy charged particles in thick absorbers, Phys. Rev. B 1, 2863-2869. 